Klaus-Uwe Panther

\title{
Der freie Dativ mir in direktiven Sprechakten
}

\begin{abstract}
Thema dieses Kapitels ist die konzeptuell-pragmatische Funktion des dativischen Personalpronomens mir in Konstruktionen mit direktivem Illokutionspotenzial. In solchen Konstruktionen erscheint mir als optionaler Dativus commodi, einer Ausprägung des ethischen Dativs. Beispiele sind Imperativsätze wie Sei mir ja vorsichtig! und syntaktisch autonome Komplementsätze wie Dass du mir ja vorsichtig bist! Das dativische Personalpronomen mir hat in solchen direktiven Sprechakten nicht seine prototypischen Eigenschaften. Es wird nicht referentiell gebraucht und fungiert nicht als Argument in der direktiven Konstruktion. Es ist jedoch konzeptuell-pragmatisch durch eine der kanonischen Funktionen des Dativs als „Nutznießer“ motiviert. Das optionale mir indiziert metonymisch sowohl eine starke attitudinale als auch eine emotive Haltung des Sprechers.
\end{abstract}

\section{Einführung}

Ziel dieses Beitrags ist es, die konzeptuell-pragmatische Funktion des freien dativischen Personalpronomens mir in Konstruktionen mit direktivem Illokutionspotenzial im Rahmen eines kognitiv-linguistischen Ansatzes zu beschreiben und zu erklären. ${ }^{1}$ Im Folgenden wird dieser freie Dativ mir - gemäß der Terminologie von Wegener (1989: 62) - als „Aufforderungs-Ethicus“ bezeichnet. Beispiele für dieses Phänomen sind Äußerungen wie Sei mir ja vorsichtig! und Dass du mir ja vorsichtig bist! Der Beitrag befasst sich vorwiegend mit Beispielen der letzteren Art, die grammatisch als unabhängige Komplementsätze kodiert werden.

Unabhängige Komplementsätze sind Instanzen des generelleren Phänomens autonomer Nebensätze, die im Deutschen und vielen anderen Sprachen

$1 \mathrm{Zu}$ „Familien“ dativischer Konstruktionen im Deutschen siehe den ausgezeichneten Überblick von De Knop \& Mollica (2017).

Mein Dank geht an die Herausgeberinnen des Bandes, Anja Binanzer, Jana Gamper und Verena Wecker, sowie interne und externe anonyme Gutachter/innen und Günter Radden für ihre sorgfältige Durchsicht des Beitrags und ihre konstruktive Kritik.

Klaus-Uwe Panther, Institut für Anglistik und Amerikanistik, Universität Hamburg 
$\mathrm{zu}$ finden sind. In Abschnitt 2 werden einige Konstruktionen dieses Typs vorgestellt, welche, obwohl sie syntaktisch wegen des fehlenden Matrixsatzes „unvollständig“ sind, Sprechakte wie Direktiva und Expressiva kodieren können. In Abschnitt 3 liegt der Schwerpunkt auf direktiv verwendeten Konstruktionen insbesondere autonomen dass-Sätzen. In Abschnitt 4 wird die semantisch-pragmatische Funktion des Aufforderungs-Ethicus mir untersucht und im Rahmen eines kognitiv-linguistischen Ansatzes die These entwickelt, dass die „Zielbedeutung“ des Aufforderungs-Ethicus mir metonymisch aus der „Quellbedeutung“ des Pronomens mir als Nutznießer einer Handlung abgeleitet werden kann. Abschnitt 5 fasst die Ergebnisse der Untersuchung zusammen und argumentiert, dass die metonymische Analyse gegenüber Ansätzen, die seinen Sinn per Implikatur ableiten, den Vorteil hat zu zeigen, dass der Aufforderungs-Ethicus kein arbiträres, sondern ein figurativ motiviertes Zeichen ist.

\section{Autonome Nebensätze mit illokutiver Funktion}

Autonome Nebensätze mit illokutiver Funktion werden von Evans (2007) als „insubordinate clauses“ bezeichnet. Der Terminus insubordinate hat im Englischen die Bedeutung ,widersetzlich, ungehorsam“ und spielt darauf an, dass sich Nebensätze unter gewissen Umständen „aufsässig“ wie Hauptsätze verhalten, d. h. sich „anmaßen“, autonome illokutive Funktionen wahrnehmen zu können. Evans (2007: 367) charakterisiert den Begriff der Insubordination folgendermaßen: „I will apply the term 'insubordination' to the conventionalized main clause use of what, on prima facie grounds, appear to be formally subordinate clauses.“ Hier sind aus einigen Sprachen Beispiele autonomer Komplementsätze, die Sprechaktfunktionen erfüllen (Panther \& Thornburg 2011: 89-90): ${ }^{2}$

\section{Englisch}

(1) That it should come to this! [Shakespeare, Hamlet, Act I, Scene 2, 137] COMP es sollte kommen zu DEM

,Dass es dazu kommen musste!‘

2 Die Abkürzungen in der Interlinearversion stehen für die folgenden grammatischen Kategorien: $\mathrm{COMP}=$ Konjunktion, die einen Komplementsatz markiert; DEM = Demonstrativpronomen; $\mathrm{GEN}=$ Genitiv; NEG = Verneinungspartikel . 


\section{Französisch}

(2) Que tout le monde sorte. [Grevisse 1993: 1561]

COMP ganze die Welt hinausgeh-SBJv

,Dass jeder hinausgehe!‘

\section{Japanisch}

(3) Saku no naka ni hair-anai koto [Okamoto 2003: 209]

Zaun GEN Mitte in betreten-NEG COMP

,Dass du nicht die umzäunte Fläche betrittst!`

\section{Deutsch}

(4) Dass es so weit kommen konnte!

Das Phänomen der Insubordination kann man auch an Konditionalsätzen beobachten, die im Deutschen und in anderen Sprachen autonom mit variierender illokutiver Kraft gebraucht werden. So kann etwa die Protasis eines Konditionalsatzes per se als Bitte oder Ausdruck der freudigen Überraschung fungieren, wie in (5) bzw. (6): ${ }^{3}$

(5) Wenn Sie etwas ruhiger sein könnten.

(6) Wenn das nicht mein alter Freund Klaus-Michael ist!

Auch autonome Komplementsätze haben im Deutschen verschiedene kommunikative Funktionen (vgl. Panther \& Thornburg 2011: 89-90):

(7) Dass du dich ja anständig benimmst!

(8) Dass doch das Semester endlich zu Ende ginge!

(9) Dass das ausgerechnet mir passieren muss!

(10) Dass ich das noch erleben darf!

Der Komplementsatz in (7) hat eine direktive Funktion, (8) drückt einen Wunsch aus, und (9) und (10) sind expressive Sprechakte, die Bedauern bzw. Freude zum Ausdruck bringen. In Abbildung 1 ist die morphosyntaktische Form und Bedeutung/Funktion direktiv gebrauchter Komplementsätze des Typs (7) schematisch dargestellt (siehe Goldberg 1995 für diese Art der Repräsentation von Konstruktionsbedeutungen).

3 Vgl. Panther und Thornburg (2005) zur illokutiven Funktion von autonom gebrauchten Protasen (if-Sätzen) im Englischen. 


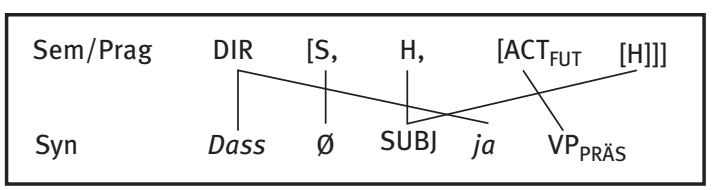

$$
\begin{array}{ll}
\text { Sem } / \text { Prag = Semantik/Pragmatik } & \text { Syn }=\text { Syntax } \\
\text { DIR = direktiver Sprechakt } & \text { Dass = Komplementierer } \\
\mathrm{S}=\text { Sprecherln } & \emptyset=\text { nicht kodiertes Element } \\
\mathrm{ACT}_{\mathrm{FUT}}=\text { zukünftige Handlung } & \mathrm{SUBJ}=\text { Subjekt } \\
\mathrm{H}=\text { Hörerln } & \mathrm{VP}_{\mathrm{PRÄS}}=\text { Verbalphrase mit Verb im Präsens }
\end{array}
$$

Abb. 1: Direktive dass-Sätze.

Auf der semantisch-pragmatischen Ebene (Sem/Prag) wird notiert, dass der Sprecher / die Sprecherin S den Hörer / die Hörerin H auffordert (DIR), eine zukünftige Handlung $\left(\mathrm{ACT}_{\mathrm{FUT}}\right.$ ) auszuführen. ${ }^{4}$ Den semantisch-pragmatischen Einheiten werden morphosyntaktische Elemente zugeordnet, die durch Linien gekennzeichnet sind. Die den autonomen Komplementsatz einleitende Konjunktion dass indiziert potentiell (aber nicht notwendig) eine direktive Funktion desselben. Diese Lesart wird durch die Partikel ja verstärkt. Der Sprecher ist nicht explizit kodiert; der Hörer des direktiven Aktes ist das grammatische Subjekt des Komplementsatzes und kann z. B. als $d u$ oder Sie realisiert werden. Der Kopf der Verbalphrase ist im Präsens kodiert, das hier auf eine zukünftige Handlung verweist.

Direktive dass-Sätze sind Konstruktionen im Sinne der Konstruktionsgrammatik. Eine Konstruktion ist ein sprachliches Zeichen, dessen Bedeutung idiomatisch ist, d. h. nicht (allein) auf der Grundlage der in ihr enthaltenen lexikalischen und grammatischen Einheiten „berechnet“ werden kann (Einleitung i. d. B.; Goldberg 2006: 5; Harnisch i.d.B.; Günthner i.d.B.). Eine Konstruktion kann also auch als ein Schema, d.h. eine Gestalt, verstanden werden, deren Bedeutung nicht aus den Bedeutungen ihrer Teile vorhergesagt werden kann (Einleitung i. d. B.; Bittner i.d. B.)

Es sei am Rande vermerkt, dass direktiv gebrauchte Komplementsätze formalen und semantisch-pragmatischen Beschränkungen unterliegen. Sie können zum Beispiel weder mit Modalverben wie können, wollen und dürfen noch mit solchen wie sollen und müssen gebraucht werden:

(11) Dass Sie ja das Fenster schließen!

(12) *Dass Sie ja das Fenster schließen können/wollen/dürfen.

(13) ${ }^{\star}$ Dass Sie ja das Fenster schließen sollen/müssen.

4 Im Folgenden werden die Termini Sprecher und Hörer für beide Geschlechter gebraucht. 
Hinsichtlich der genannten Beschränkungen kontrastieren direktive mit expressiven illokutiven Handlungen wie (9) und (10). Diese Beschränkung ist nachvollziehbar im Falle der Modalverben können, wollen und dürfen in (12), da mit ihnen keine Obligation für den Hörer ausgedrückt wird, die durch die Verbalphrase ausgedrückte Handlung auszuführen. Die Modalverben sollen und müssen in (13) denotieren jedoch eine mehr oder weniger starke Verpflichtung. Eine Erklärung für die pragmatische Inakzeptabilität von (13) ist möglicherweise, dass die Obligation des Hörers, die Handlung auszuführen, bereits in der Konstruktionsbedeutung, wie in (11), verankert ist (s. Abb. 1). Eine explizite Kodierung der Obligation durch ein Modalverb ist deshalb redundant und wird als pragmatisch abweichend empfunden.

\section{Das optionale dativische Pronomen mir in direktiven Sprechakten}

Zumindest einige Typen von direktiven Sprechaktkonstruktionen erlauben die Einfügung des dativischen Personalpronomens mir als optionales Element. Diesen Aufforderungs-Ethicus findet man beispielsweise in Imperativsätzen wie (14) und in autonomen Komplementsätzen wie (15) und (16) (Quelle: Digitales Wörterbuch der deutschen Sprache (DWDS), Webkorpus 16c):

(14) Mach mir ja keine Anstalten hier.

(15) Dass du mir ja nicht fortläufst, hast du verstanden?

(16) Dass du mir ja nicht so aufstehst!

Auch Deklarativsätze in direktiver Funktion können mit dem freien Dativ mir benutzt werden. So könnte z. B. (15) nach meinem Sprachgefühl auch als (17) formuliert werden:

(17) Du läufst mir ja nicht fort, hast du verstanden?

Selbst indirekte direktive Sprechakte (im Sinne von Searle 1975) sind offenbar mit dem freien Dativ mir kompatibel:

(18) Kannst Du mir zur Abwechslung einmal morgen pünktlich sein?

(19) Du solltest mir zur Abwechslung morgen einmal pünktlich sein! 
Der Schwerpunkt dieses Beitrags liegt auf der Verwendung des freien Dativs mir in autonomen Komplementsätzen mit direktiver Funktion. Die nachstehenden Belege für die Konstruktion sind wiederum dem Webkorpus 2016c des DWDS entnommen:

(20) Dass Sie mir ja nicht denken, Sie könnten jetzt in Rente gehen!!

(21) Dass du mir ja keine wilden Viecher anfasst!

(22) Dass du mir ja keinen von denen mit nach Hause bringst [. . . ].

(23) Und dass du mir ja nicht verschwindest in der Zwischenzeit.

(24) Und dass du mir ja wieder wohlauf zurückgebracht wirst!

Es stellt sich nun die Frage, welchen syntaktischen und semantisch-pragmatischen Status der Aufforderungs-Ethicus mir in autonomen Komplementsätzen mit direktiver Funktion hat. Ist mir ein optionales Argument des Verbs, ein optionales Argument einer Konstruktion (im Sinne von z. B. Goldberg 1995, 2006) oder etwas Drittes?

Eine Reihe von formalen Eigenschaften des Aufforderungs-Ethicus sind bereits von Wegener (1989) (vgl. auch die Zusammenfassung in Gutzmann 2007: 279-280) ermittelt worden. Zu diesen gehört unter anderem, dass der Ethicus wie eine Modalpartikel unbetont ist und nicht negiert werden kann. ${ }^{5}$ Andererseits teilt der Aufforderungs-Ethicus jedoch auch Eigenschaften mit dem dativischen Pronomen mir, das als Argument eines Verbs oder einer Konstruktion lizensiert wird. Im Unterschied zu Modalpartikeln, die in ihrer Position im Satz variabel sind, nimmt nach Gutzmann (2007) der Aufforderungs-Ethicus mir wie das Verb- oder Konstruktionsargument mir eine feste Position am linken Rand des Mittelfelds ein. Schließlich ist der Aufforderungs-Ethicus eine flektierte Form im Paradigma der Personalpronomina, während Modalpartikeln unflektiert sind. Die Tatsache, dass der Aufforderungs-Ethicus sowohl Eigenschaften von Modalpartikeln als auch solche von Personalpronomina aufweist, deutet darauf hin, dass er eine hybride Kategorie, d. h. weder ein prototypisches Exemplar der einen noch der anderen Kategorie ist. Dieser Befund ist aus kognitiv-linguistischer Perspektive nicht beunruhigend, sondern eher zu erwarten. Die Übergänge von einer grammatischen Kategorie zu einer anderen können fließend sein (siehe z. B. Taylor 2015: 569-570).

5 Das Attribut der Unbetontheit von Modalpartikeln scheint jedoch nicht uneingeschränkt zu gelten. Die Partikel ja kann durchaus prosodisch hervorgehoben werden: Dass du mir ja in Zukunft pünktlich bist!. 
Die Klärung der Frage, ob mir den Status eines optionalen Verb- oder Konstruktionsarguments hat, hängt unter anderem von Antworten auf die folgenden Teilfragen ab (siehe auch Wegener 1989; Gutzmann 2007):

1. Bezeichnet der freie Dativ mir den Sprecher?

2. Kann der freie Dativ mir mit anderen dativischen Pronomina oder Nominalphrasen koordiniert werden?

3. Kann der freie Dativ mir mit anderen Dativargumenten kontrastieren?

4. Kann der freie Dativ mir mit einem anderen nominalen oder pronominalen Dativargument innerhalb desselben (minimalen) Satzes erscheinen?

Zu Punkt 1: Die Frage, ob der Aufforderungs-Ethicus mir sich auf den Sprecher bezieht, kann mit Hilfe einer wem-Frage getestet werden. Betrachten wir einen direktiven Sprechakt wie (25):

(25) Dass du mir ja nicht die Zeit vertrödelst!

Der folgende Dialog ist pragmatisch nicht wohlgeformt, weil mir sich nicht auf den Sprecher als Person bezieht, sondern eine propositionale Einstellung und emotionale Haltung desselben induziert:

(26) A: Dass du mir ja nicht die Zeit vertrödelst!

B: *Wem (soll ich nicht die Zeit vertrödeln)?

A: ${ }^{\star}$ Mir!

Im Gegensatz zu (26) ist hingegen ein Wortwechsel wie (27) durchaus möglich, da mir als Referenten den Sprecher hat:

(27) A: Dass du mir ja morgen mein Pragmatik-Buch zurückgibst!

B: Wem (soll ich das Buch zurückgeben)?

A: Mir!

Der Sprecher will sein Pragmatik-Buch zurückerhalten; der Empfänger des Buchs ist also in diesem Fall der Sprecher selbst - nicht seine propositionale oder emotive Einstellung (obwohl mit der gegebenen Äußerung sicherlich auch zusätzlich ein Wunsch und eine emotive Haltung zum Ausdruck gebracht werden können).

Zu Punkt 2: Die These, dass mir in Äußerungen wie (25) nicht den Sprecher als Person denotiert, lässt sich auch mit der Beobachtung erhärten, dass die Koor- 
dination des freien Dativs mir mit einer dativischen Nominalphrase von zweifelhafter Akzeptabilität ist:

(28) a. Dass du mir ja nicht die Zeit vertrödelst!

b. ?'Dass du mir und meiner Schwester ja nicht die Zeit vertrödelst!

Die Konjunkte mir und meiner Schwester in (28b) sind nicht vom selben pragmatischen Typ: mir indiziert, wie bereits mehrfach betont, eine mentale und emotive Einstellung, während meiner Schwester sich auf die Schwester als Person bezieht.

Man könnte an dieser Stelle einwenden, dass der Sprecher von (28b) sich in die mentale Einstellung seiner Schwester einfühlt (Empathie), so dass meiner Schwester gegebenenfalls sich nicht auf die Schwester als Person bezieht, sondern auf ihre Wünsche und Besorgnisse hinsichtlich der zeitverschwenderischen Aktivitäten des Adressaten $d u$. Beispiele der Art (28b) kann ich allerdings empirisch nicht belegen.

Zu Punkt 3: Wenn mir den Status eines obligatorischen Verb- oder Konstruktionsarguments hat, kann es mit einem anderen Dativargument kontrastiert werden. So scheinen die folgenden Beispiele möglich:

(29) Dass du morgen ja nicht mir, sondern meiner Schwester ihr Buch zurückgibst!

(30) Dass du morgen ja nicht mir, sondern dem Geburtstagskind einen Kuchen backst!

In Beispiel (29) wird das dreistellige Verb zurückgeben in einer ditransitiven Konstruktion mit dem Rezipientenargument mir, welches den Sprecher als Person bezeichnet, verwendet. Dieses Argument wird mit dem Dativargument meiner Schwester kontrastiert. In (30) findet man den gleichen Kontrast - mit dem Unterschied, dass backen ein transitives Verb ist, aber in einer ditransitiven Konstruktion verwendet werden kann, die ein weiteres Argument verlangt, das den Rezipienten oder Nutznießer der vom Verb denotierten Handlung bezeichnet (vgl. Goldberg 1995: 9 zum englischen (to) bake ,backen').

Wenn jedoch mir als Aufforderungs-Ethicus, d.h. als Index einer mentalen Haltung und emotionalen Befindlichkeit des Sprechers benutzt wird, kann es nicht mit einer anderen dativischen Nominalphrase oder einem dativischen Pronomen kontrastiert werden:

(31) *Dass du morgen bloß nicht mir, sondern meiner Schwester / ihr pünktlich bist! 
Zu Punkt 4: Das folgende Beispiel demonstriert, dass der Aufforderungs-Ethicus mir und ein reguläres Dativargument innerhalb desselben Komplementsatzes erscheinen können:

(32) Und daß du mir ja deiner Mutter nichts anderes sagst! [Asimov 1985: 15]

Auf die Nachfrage (33a) des Adressaten der Aufforderung (32) ist (33b) eine mögliche Antwort, jedoch nicht (33c):

(33) a. Wem soll ich nichts anderes sagen?

b. Deiner Mutter!

c. ${ }^{\star}$ Mir!

Ferner kann deiner Mutter im Gegensatz zu mir mit einem anderen Dativargument kontrastiert werden. Auf die Nachfrage (34a) des Adressaten von (33) ist (34b) eine mögliche Antwort, während (34c) pragmatisch abweichend erscheint:

(34) a. Wem soll ich nichts anderes sagen?

b. Deiner Mutter - nicht deinem Vater!

c. * Mir - nicht ihr!

Die Dialoge in (33) und (34) stützen wiederum die These, dass mir in (32) eine propositionale Haltung und ein Gefühl des Sprechers ausdrückt, aber keinen Argumentstatus hat, während die Nominalphrase deiner Mutter in (32) ein vom Verb sagen lizensiertes Argument ist, dessen Referent die Mutter als Person ist.

Zusammenfassend lässt sich - im Einklang mit Wegener (1989) und Gutzmann (2007) - festhalten, dass der freie Dativ mir in direktiv gebrauchten autonomen Komplementsätzen (i) keine Antwort auf die Frage Wem? sein kann, (ii) keine Koordination mit einem dativischen Verb- oder Konstruktionsargument erlaubt und (iii) nicht kontrastiv verwendet werden kann. Fernerhin gibt es Belege, die zeigen, dass der Aufforderungs-Ethicus mir und ein dativisches Verboder Konstruktionsargument im selben Satz erscheinen können. Die genannten Eigenschaften stützen die Hypothese, dass das durch ein Verb bzw. eine Konstruktion (im Sinne von Goldberg 1995, 2006) lizensierte mir und der Aufforderungs-Ethicus mir konzeptuell und pragmatisch distinkt sind. 


\section{Semantisch-pragmatische Eigenschaften des Aufforderungs-Ethicus mir}

Meibauer (1994) und Gutzmann (2007) argumentieren, dass die Bedeutung des Aufforderungs-Ethicus mir per Implikatur abgeleitet werden kann. Meibauer (1994: 49) demonstriert seinen Ansatz mit einem Imperativsatz, der eine ditransitive Konstruktion mit dem dativischen Argument dem Papa und dem Aufforderungs-Ethicus mir enthält (meine kursive Hervorhebung):

\section{(35) Putz mir bitte dem Papa die Schuhe!}

Er rekonstruiert sodann die durch die Äußerung ausgelöste konversationelle Implikatur (36g) aus der Sicht des Hörers wie folgt (Meibauer 1994: 50; meine Nummerierung):

(36) a. Sp[recher] will, daß ich dem Papa die Schuhe putze.

b. Ich weiß, daß die Schuhe dem Papa gehören.

c. dem Papa bezeichnet den Nutznießer der geforderten Handlung.

d. mir bezeichnet normalerweise [. . .] ebenfalls den Nutznießer der Handlung.

e. Der Referent von mir kann aber nicht Nutznießer der Handlung sein, weil nicht ihm, sondern Papa die Schuhe gehören.

f. Sp hat also scheinbar gegen die Maximen der Relevanz und der Quantität verstoßen.

g. Ich kann seine Äußerung nur dann als kooperativ betrachten, wenn ich davon ausgehe, daß er mir sein besonderes Interesse am Zustandekommen der geforderten Handlung signalisieren wollte.

Eine Konversationsimplikatur im Sinne von z. B. Grice (1975) oder Levinson (2000) ist streichbar, d. h. sie kann vom Sprecher als nicht-intendierte Interpretation zurückgewiesen werden. Die Hypothese, dass der Aufforderungs-Ethicus mir eine Implikation auslöst, ist plausibel - auch wenn die involvierten inferentiellen Prozesse vollkommen spontan und automatisch ablaufen. Meibauer macht allerdings nicht deutlich, inwiefern in einer Äußerung wie (35) scheinbar gegen die Griceschen Maximen der Relevanz und der Quantität verstoßen wird (welche der beiden Quantitätsmaximen?). Ein schwerwiegenderer Einwand gegen Meibauers Analyse ist jedoch, dass der Sprecher Sp (zumindest mittelbar) genauso wie der Papa als Nutznießer der geforderten Handlung betrachtet werden 
kann. Meibauer formuliert in (36g), dass der Sprecher ein ,besonderes Interesse am Zustandekommen der geforderten Handlung“ hat. Diese Schlussfolgerung ist plausibel, aber dann liegt es nahe, nicht nur den Referenten der dativischen Nominalphrase dem Papa, sondern auch den Sprecher, der mittels mir indiziert wird, als Nutznießer der geforderten Handlung zu kategorisieren.

Gegen Meibauers (1994) Analyse argumentiert Gutzmann (2007), dass die intendierte Bedeutung von Äußerungen wie (35) per konventioneller Implikatur erschlossen wird. Gutzmann lehnt sich in seiner Konzeption an Potts (2007) an, der konventionelle Implikaturen - im Unterschied zu Grice (1975) und Levinson (2000) - als zwingende semantische Folgerungen ansieht, obwohl konventionelle Implikaturen „separate from the primary semantic content“ seien (Potts 2007: 666). Eine Konsequenz dieses Ansatzes ist, dass die vom direktiven mir ausgelöste Implikation ,Der Sprecher hat ein dringendes Interesse an der Erfüllung der geforderten Handlung،, nicht ohne Widerspruch streichbar ist.

Die von Gutzmann postulierte konventionelle Implikatur, dass der Sprecher mittels des Aufforderungs-Ethicus mir ein starkes Interesse an der Ausführung der vom Hörer geforderten Handlung bekundet, sagt nichts darüber aus, wie diese Implikation semantisch und pragmatisch $\mathrm{zu}$ erklären ist. Im Folgenden argumentiere ich, dass die in der kognitiven Linguistik entwickelten Begriffe der konzeptuellen Metonymie und des illokutiven Frames oder Szenarios (siehe z. B. Panther \& Thornburg 1998) eine relativ einfache und elegante Beschreibung und Erklärung der Bedeutung des freien Dativs mir in Direktiva erlauben. Die für die Interpretation des Aufforderungs-Ethicus mir relevante konzeptuelle Metonymie assoziiert eine Person mit ihrem Verhalten, den von ihr kreierten Produkten oder - im vorliegenden Fall - mit ihren mentalen Einstellungen und Emotionen. Diese metonymische Interpretation ist im Sinne von z. B. Pustejovsky \& Bouillon (1995) kontextuell „erzwungen“, d. h. ein Fall von „coercion“. Sie ist darüber hinaus vollkommen konventionalisiert. Eine solche erzwungene Interpretation kann nicht ohne Widerspruch zurückgenommen werden. Der von Gutzmann angenommenen konventionellen Implikatur liegt also eine konzeptuelle Metonymie zugrunde.

Der Begriff der Metonymie wird hier im Sinne von Radden \& Kövecses (2007: 336) als eine kognitive Operation verstanden, mittels derer ein konzeptuelles Vehikel (die Quellbedeutung) den mentalen Zugriff auf ein weiteres Konzept (Zielbedeutung) innerhalb desselben „idealisierten kognitiven Modells“ (engl. Idealized Cognitive Model; abgekürzt ICM) ermöglicht. Alternativ wird praktisch bedeutungsgleich - auch der Terminus Frame statt ICM benutzt, und eine Metonymie kann dann charakterisiert werden als ein Schließen von einem 
Quellkonzept auf ein mit ihm assoziiertes Zielkonzept innerhalb desselben Frames (zum Frame-Begriff in der kognitiven Linguistik siehe z. B. Ziem 2008).

Ich postuliere folglich für die Interpretation des Aufforderungs-Ethicus mir eine generische Metonymie (37a) und einen Spezialfall dieser Metonymie, nämlich (37b) (der Pfeil steht für die metonymische Beziehung zwischen Quell- und Zielbedeutung):

(37) a. Person $\rightarrow$ Mentale \& emotionale Einstellung der Person

b. Sprecher des Direktivs $\rightarrow$ Mentale \& emotionale Einstellung des Sprechers

Der Ausgangpunkt ist also die Person des Sprechers, auf die mir Bezug nimmt, aber die eigentlich intendierte und konzeptuell hervorgehobene Zielbedeutung ist die mentale Haltung und emotionale Befindlichkeit des Sprechers. Dies erklärt, warum eine wem-Frage mit der Antwort mir diskurspragmatisch unangemessen ist (siehe den Dialog (26) in Abschnitt 3).

Weiterhin ist für die Interpretation des Aufforderungs-Ethicus das illokutive Szenario für direktive Sprechakte in Abbildung 2 relevant (siehe z. B. Panther \& Köpcke 2008: 99).

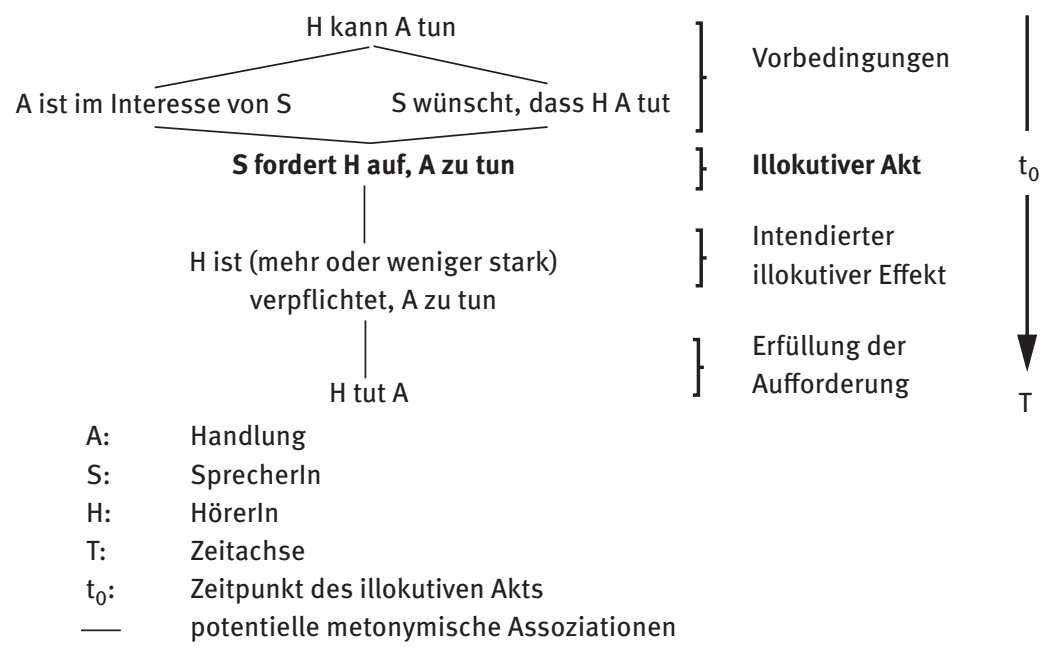

Abb. 2: Direktives Szenario.

Eine der prototypischen Bedeutungen des Dativs ist, dass der Referent der dativisch markierten nominalen oder pronominalen Einheit positiv (Benefaktiv/ Nutznießer) oder negativ (Malefaktiv) von einem Ereignis betroffen ist. In direktiven 
Sprechakten ist der Sprecher der prospektive Nutznießer der vom Hörer auszuführenden Handlung. Die im propositionalen Gehalt von Direktiva ausgedrückte zukünftige Handlung des Hörers ist für den Sprecher von Interesse, und der Sprecher wünscht, dass die entsprechende Handlung realisiert wird. Das direktive mir greift folglich auf mindestens zwei Komponenten des direktiven Szenarios zu: Interesse und Wunsch des Sprechers (s. Abb. 2).

Es kann also folgende metonymische Kette für die Interpretation des freien Dativs in Aufforderungen angesetzt werden (Abkürzungen wie in Abb. 2):

(38) a. $\operatorname{mir}(=\mathrm{S}) \rightarrow$

b. A ist im Interesse von $\mathrm{S} \rightarrow$

c. S wünscht, dass $\mathrm{H}$ A ausführt $\rightarrow$

d. S sorgt sich, das A nicht ausgeführt wird

In (38b) und (38c) werden Geglücktheitsbedingungen für direktive Sprechakte wie Bitten und Aufforderungen angesprochen. In (38b) wird das starke Interesse des Sprechers an der Realisierung der Handlung formuliert, und mit (38c) evoziert der Aufforderungs-Ethicus mir die mit direktiven Sprechakten verbundene Aufrichtigkeitsbedingung, dass der Sprecher die Realisierung der Handlung wünscht. Diese mentalen Haltungen - Interesse und Wunsch - werden also zweifach zum Ausdruck gebracht: zum einen durch den direktiven Sprechakt selbst und zum anderen durch den Aufforderungs-Ethicus mir. Der rhetorische Effekt dieser Dopplung ist eine emphatische Hervorhebung dieser beiden Einstellungen. Die Intensität des Wunsches löst schließlich noch eine weitere metonymische Assoziation aus, die in (38d) formuliert ist: Der Sprecher impliziert seine Besorgnis, dass der Hörer die geforderte Handlung nicht ausführen will und/oder wird (zur emotiven Funktion des Dativs vgl. Radden i. d. B.).

\section{Zusammenfassung und Schlussbemerkung}

In diesem Beitrag habe ich im Rahmen eines kognitiv-linguistischen Ansatzes, der illokutive Akte - hier Direktiva - als sprachliche Wissensstrukturen, d. h. als Frames, auffasst, argumentiert, dass die Zielbedeutung des Aufforderungs-Ethicus mir mittels einer Kette von metonymischen Schlussfolgerungen beschrieben werden kann. Konzeptuelle Metonymien nutzen assoziative Beziehungen zwischen Frame-Komponenten, um Zielbedeutungen zu kreieren. Im vorliegenden Fall ist der ,Sprecher als Person“ der Ausgangspunkt für die Ableitung der Zielbedeutung ,mentale Haltung und emotionale Befindlichkeit des Sprechers`. Diese 
Zielbedeutungen, die nicht auf den Sprecher selbst, sondern auf seine mentale Haltung (Wunsch) und sein Gefühl (Besorgnis) Bezug nehmen, erklären, warum der Aufforderungs-Ethicus mir keine Antwort auf die Frage Wem? sein kann, weil diese Frage normalerweise mit einer Personenbezeichnung beantwortet wird nicht mit einem Ausdruck, der eine propositionale Einstellung oder Emotion der betreffenden Person ausdrückt. Es sind die Zielbedeutungen des AufforderungsEthicus mir, die das ihm eigene formale Verhalten erklären, welches mit dem normalen Gebrauch des Personalpronomens mir als Verb- oder Konstruktionsargument kontrastiert.

Zusammenfassend lässt sich sagen, dass in seiner prototypischen Verwendung als Verb- oder Konstruktionsargument das dativische Personalpronomen mir folgende Eigenschaften aufweist: Wie in Abschnitt 3 gezeigt, ist es

1. ein sprachliches Zeichen mit referentieller Funktion,

2. ein obligatorisches oder optionales Argument einer Konstruktion oder eines Verbs,

3. und es ist durch semantische Rollen wie ,Nutznießer` und ,Rezipient` gekennzeichnet.

Im Gegensatz zu seiner prototypischen Verwendung als Bezeichnung des Sprechers weist mir im Aufforderungs-Ethicus folgende Attribute auf:

1. Es ist kein referentieller Ausdruck, sondern impliziert metonymisch die mentale und emotionale Haltung des Sprechers.

2. Es ist ein optionales Element und hat keinen Status als Argument einer Konstruktion oder eines verbalen Ausdrucks.

3. Es kann mit einem dativischen Argument im selben Satz kollokieren.

Die Frage, ob der Aufforderungs-Ethicus ein Personalpronomen oder eine Modalpartikel ist, kann also nicht mit einem klaren Ja oder Nein beantwortet werden. Der Aufforderungs-Ethicus mir ist prototypisch weder ein Personalpronomen noch eine Modalpartikel mit direktiver Funktion, sondern eine Kategorie, die peripher sowohl Eigenschaften der einen als auch der anderen Kategorie aufweist.

Schließlich hat die metonymische Zerlegung des Aufforderungs-Ethicus ergeben, dass er kein arbiträres sprachliches Zeichen im Sinne von Ferdinand de Saussure ist - im Gegenteil, seine Bedeutung und sein Gebrauch sind durch die Basisbedeutung des dativischen Personalpronomens mir motiviert. 


\section{Literatur}

Binanzer, Anja, Jana Gamper \& Verena Wecker (i. d. B.): Einleitung.

Bittner, Dagmar (i. d. B.): Strukturalistische vs. gebrauchsbasierte Modellierung des Erwerbs der definiten Artikel und D-Pronomen des Deutschen.

De Knop, Sabine \& Fabio Mollica (2017): The family of German dative constructions. In Francisco J. Ruiz de Mendoza Ibáñez, Alba Luzondo Oyón \& Paula Pérez Sobrino (Hrsg.), Constructing families of constructions: Analytical perspectives and theoretical challenges (Human Cognitive Processing 58), 205-238. Amsterdam, Philadelphia: John Benjamins.

Evans, Nicholas (2007): Insubordination and its uses. In Irena Nikolaeva (Hrsg.), Finiteness: Theoretical and empirical foundations, 366-431. Oxford: Oxford University Press.

Goldberg, Adele E. (1995): Constructions: A construction grammar approach to argument structure. Chicago, London: University of Chicago Press.

Goldberg, Adele E. (2006): Constructions at work: The nature of generalization in language. Oxford: Oxford University Press.

Grevisse, Maurice (1993): Le bon usage: Grammaire française refondue par André Goosse. Paris, Louvain-la-Neuve: Éditions Duculot.

Grice, H. Paul (1975): Logic and conversation. In Peter Cole \& Jerry Morgan (Hrsg.), Speech acts (Syntax and Semantics 3), 41-58. New York: Academic Press.

Günthner, Susanne (i. d. B.): Wenn-Konstruktionen im Gespräch: Zur Verwobenheit kognitiver und interaktionaler Faktoren bei der Realisierung grammatischer Muster.

Gutzmann, Daniel (2007). Eine Implikatur konventioneller Art: Der Dativus Ethicus. Linguistische Berichte 211, 277-308.

Harnisch, Rüdiger (i. d. B.): Von weiblichen Leserinnen und Frauenskispringerinnen: Tautologische Syntagmen auf dem Weg zu festen Konstruktionen.

Levinson, Stephen C. (2000): Presumptive meanings: The theory of generalized conversational implicature. Cambridge, MA: MIT Press.

Meibauer, Jörg (1994): Modaler Kontrast und konzeptuelle Verschiebung. Studien zur Syntax und Semantik deutscher Modalpartikeln. Tübingen: Niemeyer.

Okamoto, Shigeko (2003): Metonymy and pragmatic inference in the functional reanalysis of grammatical morphemes in Japanese. In Klaus-Uwe Panther \& Linda L. Thornburg (Hrsg.), Metonymy and pragmatic inferencing (Pragmatics \& Beyond New Series 113), 205-220. Amsterdam, Philadelphia: John Benjamins.

Panther, Klaus-Uwe \& Klaus-Michael Köpcke (2008): A prototype approach to sentences and sentence types. Annual Review of Cognitive Linguistics 6, 83-112.

Panther, Klaus-Uwe \& Linda L. Thornburg (1998): A cognitive approach to inferencing in conversation. Journal of Pragmatics 30, 755-769.

Panther, Klaus-Uwe \& Linda L. Thornburg (2005): Motivation and convention in some speechact constructions: A cognitive linguistic approach. In Sophia Marmaridou, Kiki Nikiforidou \& Eleni Antonopoulou (Hrsg.), Reviewing linguistic thought: Converging trends for the $21^{\text {st }}$ century (Trends in Linguistics: Studies in Monographs 161), 53-78. Berlin, New York: De Gruyter Mouton.

Panther, Klaus-Uwe \& Linda L. Thornburg (2011): Emotion and Desire in independent complement clauses: A case study from German. In Mario Brdar, Milena Žic Fuchs \& Stefan T. Gries (Hrsg.), Converging and diverging tendencies in cognitive linguistics (Human Cognitive Processing 32), 87-114. Amsterdam, Philadelphia: John Benjamins. 
Potts, Christopher (2007): Into the conventional-implicature dimension. Philosophy Compass 2, 665-679.

Pustejovsky, James \& Pierrette Bouillon (1995): Aspectual coercion and logical polysemy. Journal of Semantics 12, 133-162.

Radden, Günter (i. d. B.): Der verkannte $e$-Dativ im heutigen Deutsch: im Kreise meiner Lieben und am Rande des Abgrunds.

Radden, Günter \& Zoltán Kövecses (2007): Towards a theory of metonymy. In Vyvyan Evans, Benjamin K. Bergen \& Jörg Zinken (Hrsg.), The cognitive linguistics reader, 335-359. London, Oakville: Equinox.

Searle, John R. (1975): Indirect speech acts. In Peter Cole \& Jerry Morgan (Hrsg.), Speech acts (Syntax and Semantics 3), 59-82. New York: Academic Press.

Taylor, John (2015): Prototype effects in grammar. In Ewa Dąbrowska \& Dagmar Divjak (Hrsg.), Handbook of cognitive linguistics (HSK 39), 562-579. Berlin, Boston: De Gruyter.

Wegener, Heide (1989): Eine Modalpartikel der besonderen Art: Der Dativus Ethicus. In Harald Weydt (Hrsg.), Sprechen mit Partikeln, 56-76. Berlin, New York: De Gruyter.

Ziem, Alexander (2008). Frames und sprachliches Wissen: Kognitive Aspekte der semantischen Kompetenz. Berlin, New York: De Gruyter.

\section{Quellennachweise}

Asimov, Isaac (1985): Aurora oder der Aufbruch zu den Sternen. München: Heyne, S. 15.

Die Belege (14)-(16) und (20)-(26) sind dem Webkorpus 16c des Digitalen Wörterbuchs der deutschen Sprache (DWDS) entnommen: https://www.dwds.de/d/k-web\#ibk_web_2016c (abgerufen am 25.05.2020). Die übrigen Beispiele stammen aus der Fachliteratur oder sind introspektiv gebildet. 